\title{
WARP-KNITTED FABRIC DEFECT SEGMENTATION BASED ON NON-SUBSAMPLED WAVELET-BASED CONTOURLET TRANSFORM
}

\author{
Dong Xia, Gaoming Jiang*, Pibo Ma \\ Engineering Research Center for Knitting Technology, Jiangnan University , Jiangsu, Wuxi, 214122, China \\ *Corresponding author E-mail: Jiang G. jgm@jiangnan.edu.cn
}

\begin{abstract}
:
In this paper, a non-subsampled wavelet-based contourlet transform (NWCT) is applied in warp-knitted fabric defect segmentation. Compared with the traditional contourlet transform, wavelet transform takes the place of Laplacian pyramid in NWCT and the directional filter bank is non-subsampled. The wavelet transform with improved wavelet threshold is put to use, and the original fabric image can be decomposed into low-frequency approximate coefficient $A$ and high-frequency detail coefficients $\mathrm{V}, \mathrm{H}$, and $\mathrm{D}$. The high-frequency detail coefficients are processed by the non-subsampled directional filter bank to get directional sub-band coefficients. Afterward, the effective sub-band coefficients based on regional energy are chosen to reconstruct $\mathrm{V}, \mathrm{H}$, and $\mathrm{D}$. And the reconstructed fabric image will be achieved by inverse non-subsampled wavelet-based contourlet transform. The adaptive threshold method and morphological processing are used to obtain the legible defect profile. The experiment demonstrates that NWCT can achieve the positive segmentation regarding the common defects, such as broken warp, width barrier, and oil, and has excellent performance on these directional defects and regional defects. It is acknowledged that NWCT will provide a new way to detect warp-knitted fabric defects automatically.
\end{abstract}

\section{Keywords:}

Warp-knitted fabric defect; non-subsampled wavelet-based contourlet transform (NWCT); non-subsampled directional filter

\section{Introduction}

Following the digitization of the textile industry, emphasis is on the manufacturing process automation overwhelmingly. Accordingly, it is universally understood that the fabric defect is behind the times and unsuitable for the modern textile industrial production [1]. In addition, the automatic detection of fabric defect is capturing the researchers' attention and it is more accurate and efficient. Nevertheless, much of the research in automatic detection of fabric defect in the last decades has examined in the field of woven fabric, especially the plain one, and the work in the warp-knitted fabric is unusual. Alternatively, the automatic detection of fabric defect is mainly dependent upon spatial domain methods (gray histogram statistics method, gray level co-occurrence matrix [2-4], and Markov random field method [5-6]) or frequency domain methods (Fourier transform method [7-9], Gabor transform [10-12], and wavelet transform [13-15]). However, these methods fail to effectively capture the information on the fabric defect.

In this work, the non-subsampled wavelet-based contourlet transform (an improved contourlet transform; NWCT) is applied in the warp-knitted fabric defect segmentation for the first time. Different from the traditional contourlet transform proposed by Do and Vetterli [16-17], NWCT substitutes wavelet transform for Laplacian pyramid to obtain the high-frequency detail coefficients and provides the non-subsampled directional filter bank to decompose these coefficients. Similar to contourlet transform, it also can play an important role in the area of image compression, image fusion, and image de-noising. This paper takes the loop structure of warp-knitted fabric into consideration and segments the broken warp, width barrier, and oil produced in the warp-knitting production out of the fabric. The experimental results imply that the new method gives an outstanding performance in profile-holding ability.

\section{Contourlet transform}

It is well known that Fourier transform reveals the inherent relationship between the time function and spectrum function. It displays the whole spectrum of the entire time period. Although Fourier transform has the excellent performance of frequency localization, it fails to achieve time localization. As known, the time localization is crucial to processing the nonstationary signal. However, the wavelet transform, which has the advantage of time-frequency localization, can address the shortcoming. The wavelet transform can provide time-frequency analyses for the input signal. But when the input signal contains highdimensional singular geometrical characteristics, such as edge, profile, texture, and so on, the wavelet transform is no longer applicable for the optimal approximation of the original signal. Afterward, some researchers proposed a significant amount of improved wavelet or Fourier methods and these methods also achieved some anticipated goals, but the inherent matter still exist. Naturally the multiscale geometric analysis, which is a proactive response to these matters, is proposed. Among them, the contourlet transform is one of excellent geometrical 
image transforms that can decompose images both radially and angularly while keeping the favorable profile. It can hold much more high-dimensional singular geometrical characteristics so that to extract the intrinsic geometric features of images and get the "real" 2-D images. Thus it is widely shared that the contourlet transform is playing a more important role in the fields of image compression, image de-noising, image fusion, and texture analysis [18].

Generally, the contourlet transform consists of Laplacian pyramid (LP) and directional filter bank (DFB, proposed by Bamberger and Smith in 1992 [19]). Figure 1 shows the operating principle of the contourlet transform. In the figure, the original image is decomposed into low-frequency coefficient and high-frequency coefficient by LP [20] and then the highfrequency coefficient continues to be decomposed radially and angularly by DFB. Consequently, the directional sub-bands will be formed.

Shown in Figure 2 is the theory of LP decomposition. In this, Sig means the input signal; $D$ is decomposition filter; $S$ represents the synthesis filter; $D S M$ is down-sampled matrix; and USM represents up-sampled matrix. The original signal Sig is filtered by $D$ and sampled by $D S M$. It will produce the low-frequency signal $a$. This signal continues to be operated by means of $S$ and USM, and a prediction signal, which is similar to the original signal, is formed. In the end, the high-frequency signal $b$ is worked out, which is equal to the $D$-value between the original signal and prediction signal.

Moreover, the DFB is a tree-structure of $N$ levels that can decomposes the high-frequency coefficient into directional sub-bands at every level [21]. And the DFB is mainly composed of the two-band fan filter bank, the following sampled matrix $Q 1$ and $Q 2$ and the sampled unimodular matrix $R 1, R 2, R 3$, and $R 4$.

$$
\begin{gathered}
Q 1=\left[\begin{array}{cc}
1 & -1 \\
1 & 1
\end{array}\right], Q 2=\left[\begin{array}{cc}
1 & 1 \\
-1 & 1
\end{array}\right] \\
R 1=\left[\begin{array}{ll}
1 & 1 \\
0 & 1
\end{array}\right], R 2=\left[\begin{array}{rr}
1 & -1 \\
0 & 1
\end{array}\right], R 3=\left[\begin{array}{ll}
1 & 0 \\
1 & 1
\end{array}\right], R 4=\left[\begin{array}{cc}
1 & 0 \\
-1 & 1
\end{array}\right]
\end{gathered}
$$

The fan filter bank just filters the frequency spectrum in both the horizontal and vertical directions. However, the down-sampled matrix changes the data sampling rate and rearranges the result while the down-sampled unimodular matrix merely rearranges the result. In general, the decomposition steps are manifest as in Figure 3. In the figure, $D Q 1$ and $D Q 2$ are respectively downsampling operation of $Q 1$ and $Q 2$, and $D R 1, D R 2, D R 3$, and DR4 are same too.

\section{Non-subsampled wavelet-based contourlet}

\section{transform}

Although the contourlet transform can hold the profile information of the original image positively, it is not better than the wavelet transform with regard to the total effect. There are several reasons behind this case. In the first place, LP forms the heart of the matter. As mentioned above, the contourlet transform is composed of LP and DFB. For LP, there is an original image waiting for decomposition at the first level and an approximate coefficient at the last level. The resolution and size of the image will fall off when the decomposition begins.

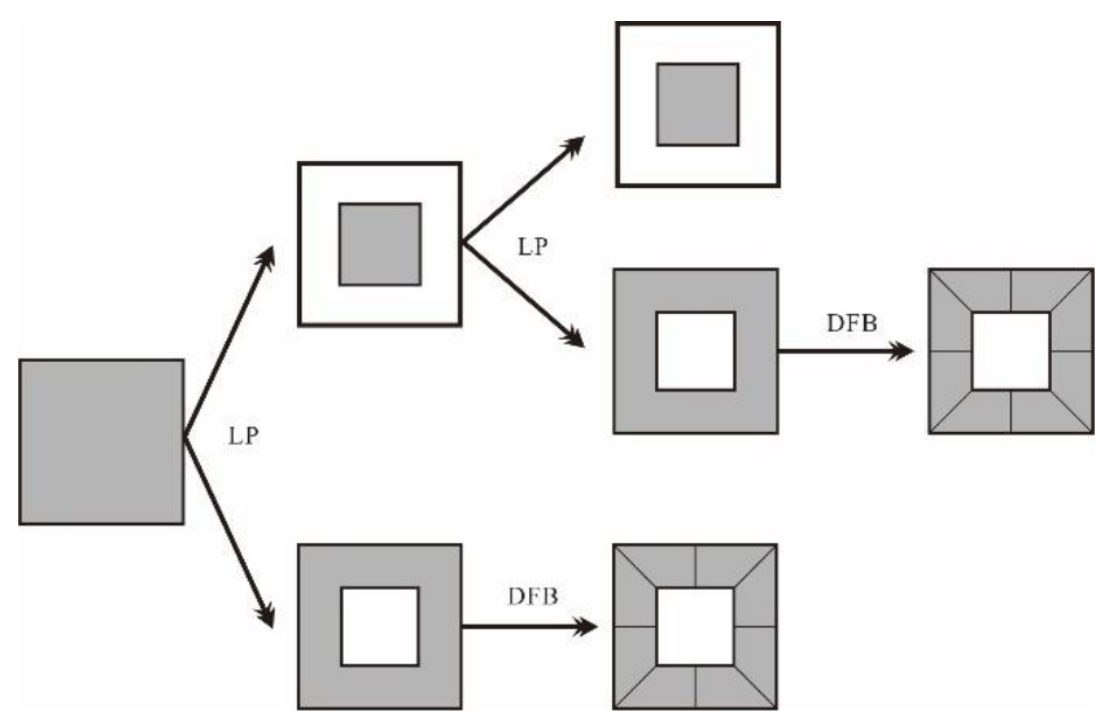

Figure 1. The operating principle of the Contourlet transform

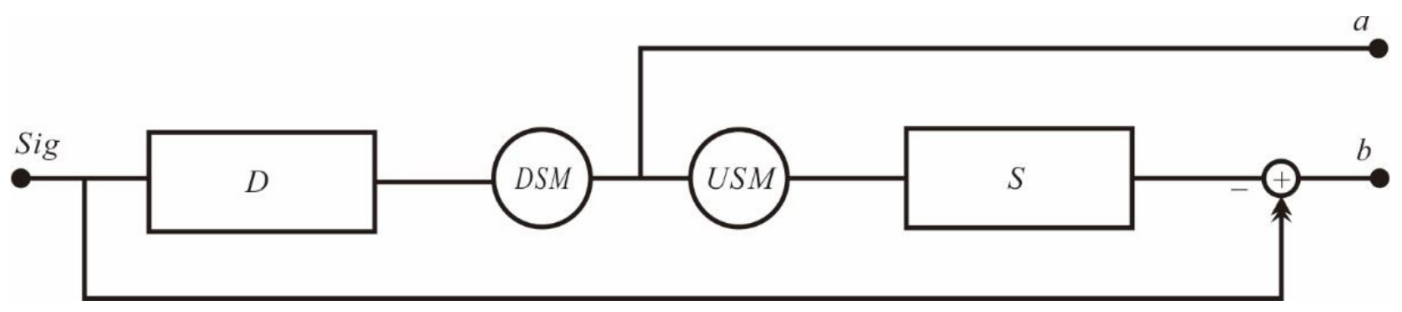

Figure 2.The LP decomposition 


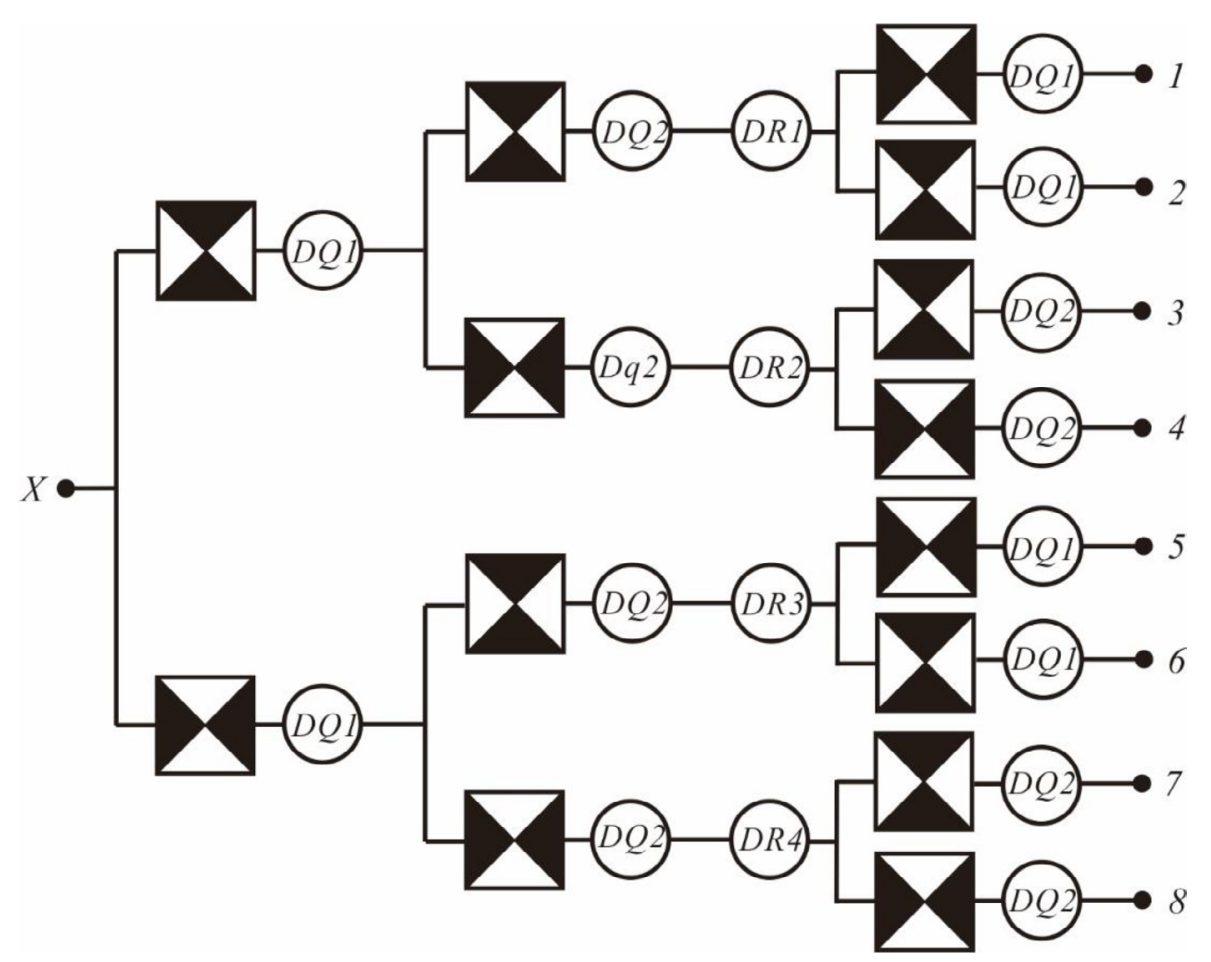

Figure 3.The decomposition steps of DFB

For example, there is an image of size $2^{\jmath *} 2^{J}$ (equals to $N^{*} N$, $\left.J=\log _{2} N\right)$. After the decomposition of $P+1$ levels, the resolution of it will turn into $2^{J-P *} 2^{J-P}$. Consequently, $M$ pixel of $P+1$ levels has the following formula:

$$
N^{2}\left[1+\frac{1}{(4)^{1}}+\frac{1}{(4)^{2}}+\cdots+\frac{1}{(4)^{P}}\right] \leq \frac{4}{3} N^{2}
$$

It can be found that LP will produce the redundancy of $1 / 3$. Accordingly, it leads to the redundancy of the contourlet transform. As a result, the redundancy will give rise to jumbled information and the calculated amount of the transform increases. So the wavelet transform is introduced to substitute LP [22].

Compared with Fourier transform, wavelet transform (developed by Meyer and Mallat) is a local transformation based on time and frequency domains so that it can extract the information from the input signal effectively. It can achieve multiscale analysis through scale and translation transformation and solve matters in which the Fourier transform failed. So when the wavelet transform substitutes for LP to decompose the image, it can settle the matter of redundancy and reduce the calculated amount of the whole transform [23-25].

As shown in Figure 4, 2-D wavelet transform decomposes the image into low-frequency coefficient $C$ and high frequency coefficients $H, V$, and $D$. And the equations of the coefficients are respectively as follows.

$$
\begin{gathered}
W_{\varphi}^{C}\left(p_{0}, m, n\right)=\frac{1}{\sqrt{M N}} \sum_{x=0}^{M-1} \sum_{y=0}^{N-1} f(x, y) \varphi_{p_{0}, m, n}(x, y) \\
W_{\psi}^{i}(p, m, n)=\frac{1}{\sqrt{M N}} \sum_{x=0}^{M-1} \sum_{y=0}^{N-1} f(x, y) \psi_{p, m, n}^{i}(x, y), i=\{H, V, D\}
\end{gathered}
$$

where $f(x, y)$ is a 2-D image; $\varphi(x, y)$ and $\psi(x, y)$ represent scale function and wavelet function respectively; and generally $p_{0}$ is valued as $0, M=N=2^{p}, p=0,1,2, \ldots, J-1$, and $m=n=0,1,2, \ldots, 2^{j}$ -1 .

One more point needs to be described. In order to solve this problem, Eslami has proposed wavelet-based contourlet transform over the years, but the actual effect is not promising [26-27]. The reason for that is the artifacts it generates. Actually, the traditional directional filter bank, which is operated with down-sampled matrix, destroys the translation invariance and results in artifacts. Especially, the artifacts will be distinguished when the image has dramatic changes of the gray level. Similarly, the gray level changes rapidly as the defect occurs on the fabric, and accordingly the artifacts will be almost certainly drawn into the defect segmentation.

So it is indispensable to discuss about the down-sampled matrix. This paper defines $M$ as a sampled matrix, $x(n)$ as the original signal, and $X(\omega)$ as its spectrum. The procedure of down-sampling is demonstrated as follows.

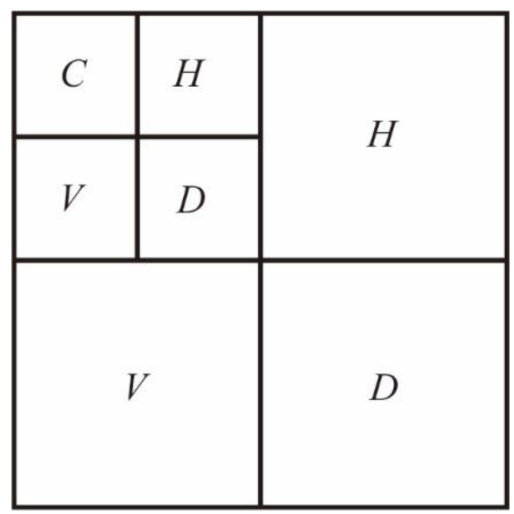

Figure 4. The schematic diagram of Wavelet transform 


$$
\begin{aligned}
& x_{D S}(n)=x(M n) \\
& X_{D S}(\omega)=\frac{1}{M} \sum_{k=0}^{M-1} X\left(\frac{\omega-2 k \pi}{M}\right)
\end{aligned}
$$

Usually, we adopt 2 as determ of sampled matrix $M$. According to equation (7), the new expression looks like equation (8).

$$
X_{D S 2}(\omega)=\frac{1}{2}\left[X\left(\frac{\omega}{2}\right)+X\left(\frac{\omega}{2}-\pi\right)\right]
$$

As above, the highest frequency is doubled, which means the down-sampling operation expands the spectrum of the input signal. Naturally, the down-sampling operation will give rise to the aliasing of the signal if the bandwidth of the signal is sufficient. That explains the reason of why the DFB is unsuitable. Figure 6 also demonstrates the procedure of downsampling.
However, the non-subsampled directional filter bank (NSDFB) can be constructed if the down-sampling operation is replaced by up-sampling operation [28]. Shown as the following equations, the up-sampling matrix does not lead to the aliasing of the signal, so it is promising enough to construct NSDFB.

$$
\begin{aligned}
& x_{U S}(n)=x\left(M^{-1} n\right) \\
& X_{U S}(\omega)=X(M \omega)
\end{aligned}
$$

As shown as Figure 6, it's the non-subsampled directional filter banks that is constructed in this work. The down-sampling operation is removed. In contrast, the up-sampling operation is introduced. The first level is the fan filter bank. When the fan filters are up-sampled by the sampled matrix $Q 1$ and $Q 2$, the second level filters are formed, which are known as

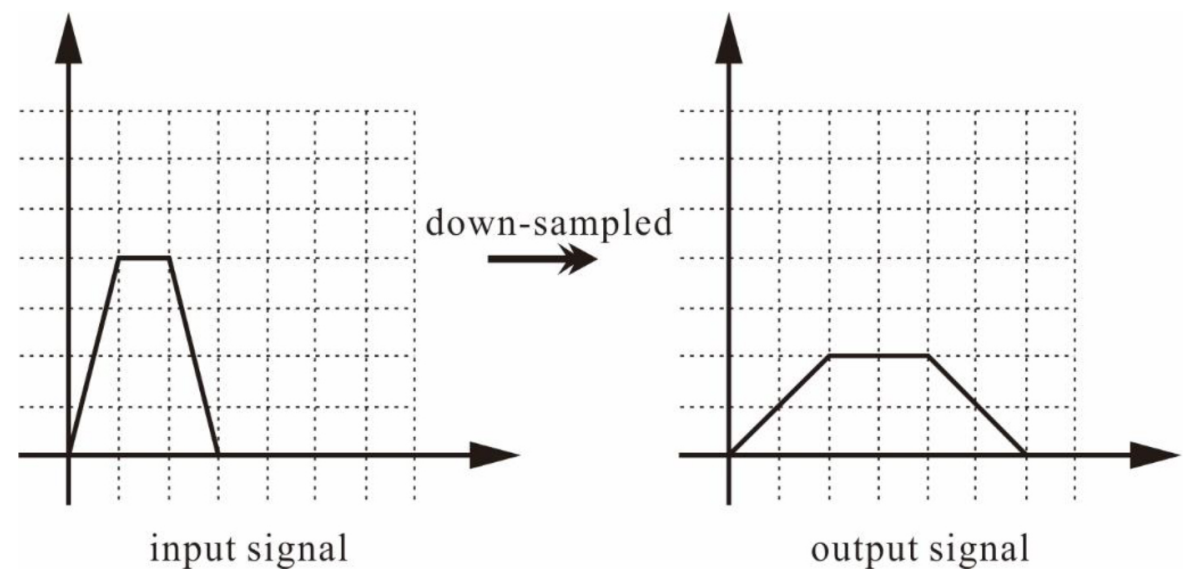

Figure 5. The down-sampling operation

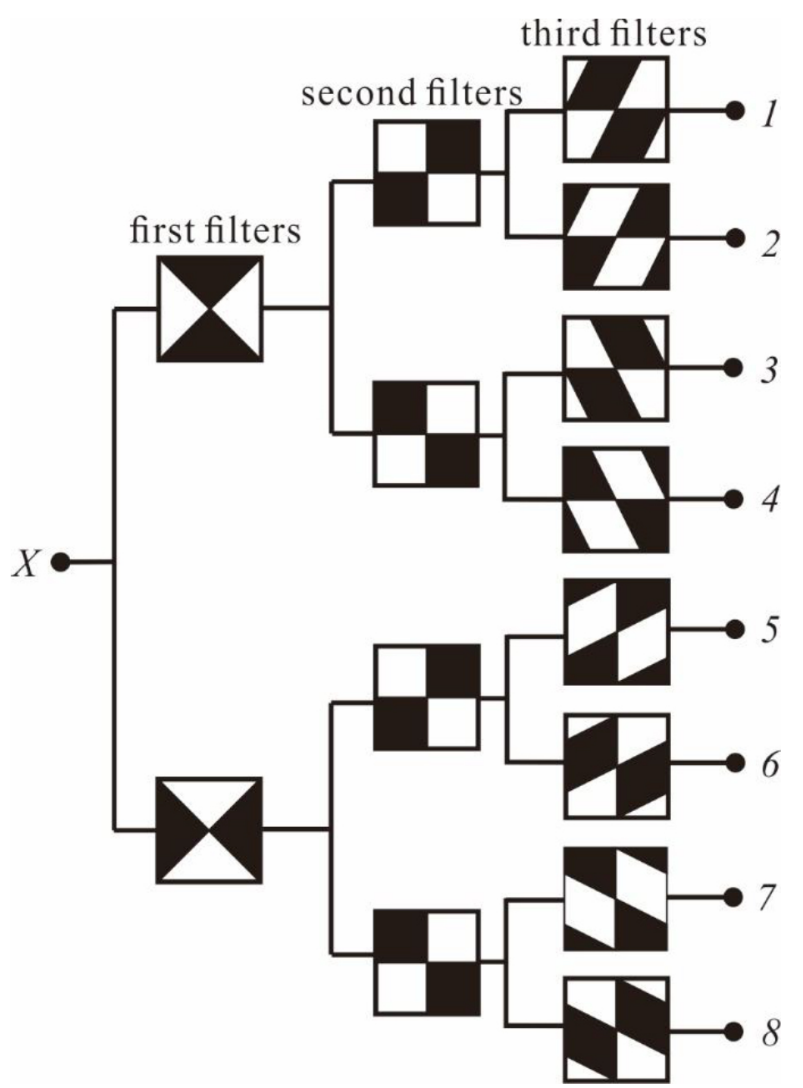

Figure 6.The decomposition steps of NDFB 
checkerboard-shaped filters. The checkerboard-shaped filters cascading the first level fan filters can achieve the four-band decomposition. Similarly, the third filters (the parallelogram filters) will be constructed when the fan filters are up-sampled by the unimodular matrix $R 1, R 2, R 3$, and $R 4$. This can result in the eight-band decomposition of the input signal.

In general, the contourlet transform is improved by wavelet transform and the non-subsampled directional filter bank. The new one (non-subsampled wavelet-based contourlet transform) overcomes the disadvantages of the contourlet transform and will have the broader way for image processing. Figure 7 shows the decomposition procedure of NWCT.

\section{Defect segmentation}

\subsection{Decomposition}

As shown in Figure 8, it's a warp-knitted fabric image included broken warp which is of size $256 \times 256$ and it is captured by EPSON V370 Photo. This fabric sample used in the paper is the one of warp-knitted fabric produced on high-speed Tricot machine. In addition, it is made of polyester filament yarn and its loop structure image is shown in Figure 14, in which it is respectively the technical face image and technical back image. During the decomposition, the fundamental wave for wavelet transform and NSDFB have to be taken into consideration. As known, the gray level of the fabric image will change rapidly while the defect emerges. Moreover, the spatial distribution of the gray level is peak shaped. As Figure 9 demonstrates, the bior2.8 is peak shaped too and is similar to fabric image graylevel changes.

In the next moment, the fundamental wave of NSDFB should be decided. Allow for the biorthogonality of bior2.8, the series of pkva wavelet and 9/7 wavelet function are compatible with it. In order to select the best one, this paper takes the information entropy into account. The computational formula of the information entropy is manifest as following.

$$
H=\sum_{i=0}^{255} G_{i} \log _{2} G_{i}
$$

Here, $G_{i}$ represents the occurrence probability of gray level $i$.

In the experiment, the information entropy of the four directional sub-bands regarding to the broken warp fabric, width barrier fabric, and oiled fabric are compared. The sub-band that produces the maximum is chosen as the best suitable fundamental wave. Because the larger the information entropy, the more the profile information, and the more suitable the fundamental wave. In Table 1, the information entropies of the broken warp fabric (vertical), width barrier fabric (horizontal), and oiled fabric (all directions) are listed and these apparently demonstrate that pkva6 is the best one for the NSDFB.

However, this paper also adopts an improved wavelet threshold denoising method during the decomposition of the wavelet transform. The traditional approaches concerning the wavelet threshold denoising are hard threshold and soft threshold functions. But these methods will have an impact on the integral continuity of the processed coefficients. So this work improves the method by introducing the weighted average method:

$\operatorname{coe}_{i, j}=\left\{\begin{array}{c}(1-w) * \operatorname{coef}_{i, j}+w *\left(\operatorname{coe}_{i, j}-\lambda\right),\left|\operatorname{coe}_{i, j}\right| \geq \lambda \\ 0, \\ \left|\operatorname{coe} f_{i, j}\right|<\lambda\end{array}\right.$

$$
w=\frac{\lambda}{\left|\operatorname{coef}_{i, j}\right| * \exp \left(\sqrt{\mid \frac{\left|\operatorname{coef}_{i, j}\right|-\lambda}{\left|\cos _{i, j}\right|+\lambda}}\right)}, \quad \lambda=\sigma \sqrt{2 \log N}
$$

Here, $\sigma$ is the noise standard deviation and $N$ is the decomposition level; $\lambda$ and $w$ is respectively the threshold and the weight; $\operatorname{coef}_{i, j}$ represents the gray value of the coefficients $V, H$, and $D$.

Meanwhile, the level of decomposition should be also considered. The ringing and profile information are the crucial factors to determine the level. In the paper, the sum of the energy of the high-frequency coefficients is calculated at the $N$

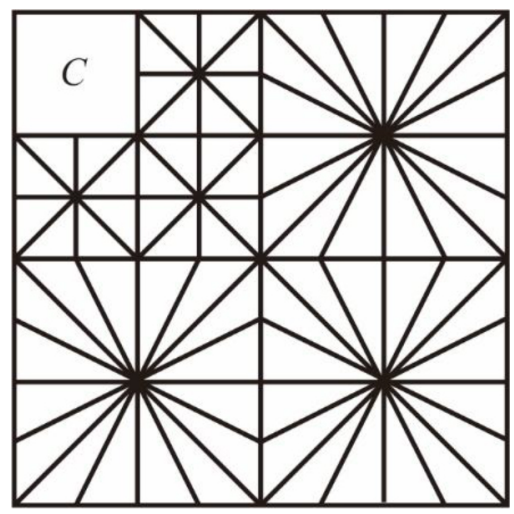

Figure 7. The decomposition of NWCT

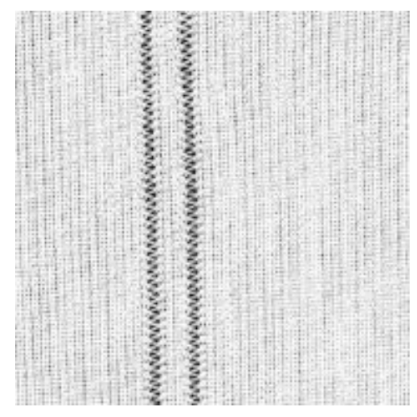

Figure 8. The warp-knitted fabric with broken warp

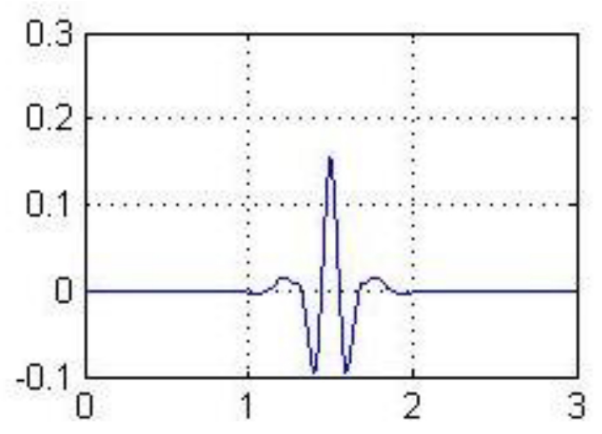

Figure 9. The fundamental wave bior2.8 
Table 1. The information entropy of the fabric defect directional sub-bands

\begin{tabular}{|c|c|c|c|c|c|}
\hline \multicolumn{2}{|c|}{ Kind of fabric defect } \\
\hline \multirow{2}{*}{$\begin{array}{c}\text { Fundamental } \\
\text { wave }\end{array}$} & Broken warp & Width barrier & & \multicolumn{2}{c|}{ Oil } \\
\cline { 2 - 6 } & Vertical & Horizontal & Vertical & 3.3039 & 2.5527 \\
\hline $9 / 7$ & 3.5138 & 2.9665 & 3.8008 & 3.4586 & 2.6232 \\
\hline pkva & 3.5624 & 3.0694 & 3.9548 & 3.6035 & 2.8165 \\
\hline pkva6 & 3.6087 & 3.1139 & 4.0055 & 3.5144 & 2.7203 \\
\hline pkva8 & 3.5793 & 3.0812 & 3.9601 & & Diagonal \\
\hline
\end{tabular}

level and then divided by the last level. If the $D N>1$, the image should be decomposed again; if not, the now level is the best. And in the paper, the best level is 2 .

$$
D_{N}=\frac{E_{N}^{V}+E_{N}^{H}+E_{N}^{D}}{E_{N-1}^{V}+E_{N-1}^{H}+E_{N-1}^{D}}
$$

Here, $\mathrm{E}_{\mathrm{N}}^{\mathrm{i}}(i=V, H, D)$ means the energy of the high-frequency coefficients at the $N$ level. Consequently, Figure 10 to 12 show the results regarding the decomposition of the broken warp fabric.

\subsection{Reconstruction by regional energy}

After the decomposition, the image is divided into low frequency coefficient and directional sub-bands and these sub-bands present the profile information of the image. Then choose the promising sub-bands for the profile information based on regional energy. The theory of the process is as follows, here, $\mathrm{M}_{\mathrm{N}}^{\mathrm{k}}$ is the $k \mathrm{st}(k=1,2 \ldots 2 N+1)$ directional sub-band matrix at the $N$ th level of the image; and $W^{k}(n, n)$ is the weighting coefficient corresponding to $\mathrm{M}_{\mathrm{N}}^{\mathrm{k}} ; n$ is 3 or 5 .

$$
E_{N}^{k}(x, y)=\sum_{n} W^{k}(n, n)\left[M_{N}^{k}(x+n, y+n)\right]^{2}
$$

Then choose the promising sub-bands of these high-frequency coefficients. Usually, the promising sub-bands of coefficient $V$ is vertical sub-bands meanwhile coefficient $H$ is horizontal and coefficient $D$ is the maxima one. Then the high-frequency coefficients $V, H$, and $D$ will be reconstructed by the inverse derivation of NSDFB and the fabric image will be also reconstructed based on the invert wavelet transform. Figure 13 shows the reconstructed fabric image of the broken warp fabric. It keeps most of the information of the picture.

\subsection{Adaptive threshold and morphological opening}

Shown in Figure 14 is the loop structure of the warp-knitted fabric sample. According to the definition of the warp-knitted fabric, it's the fabric that makes the yarn bent to loop and draw the new loop through the old loop [29]. It can give rise to the hole effect, especially in the vertical direction so that result in the gray level changes like the defect. Consequently, the last binary image segmented by the single threshold is filled with a host of unpromising noise. However, the adaptive threshold can resolve this matter. For the theory of it, the adaptive threshold provides a series of thresholds instead to segment different parts of images and it has excellent anti-noise property when the image is of uneven illumination or burst noise. But the image remains to be processed by means of morphological opening to smooth the segmented result. The principle of morphological opening is image erosion and image dilation, which erases the tiny objects

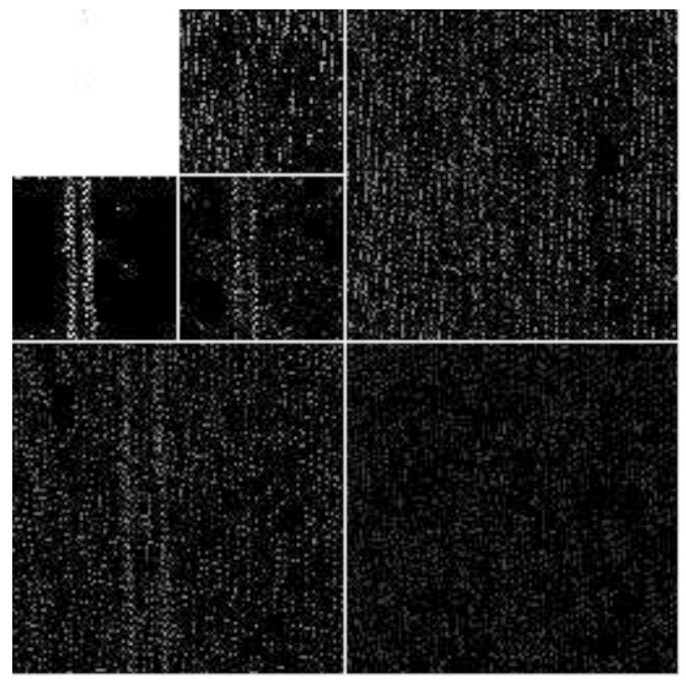

Figure 10. The decomposition of wavelet transform
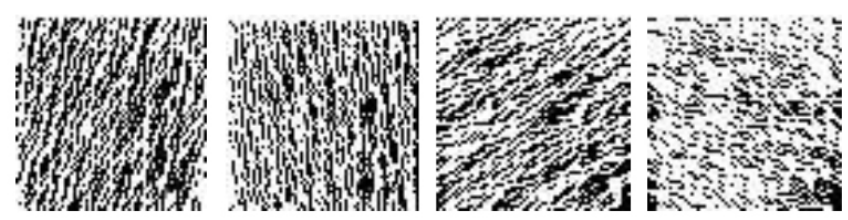

(a) The sub-bands of horizontal direction
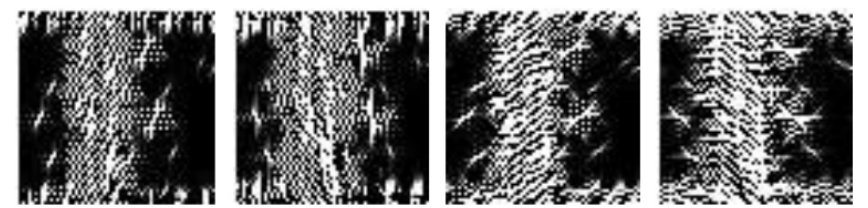

(b) The sub-bands of vertical direction
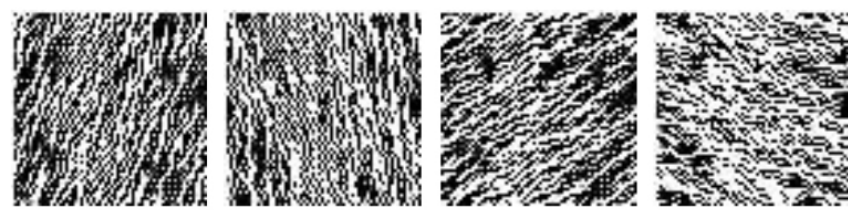

(c) The sub-bands of diagonal direction

Figure 11. 4 directional sub-bands 

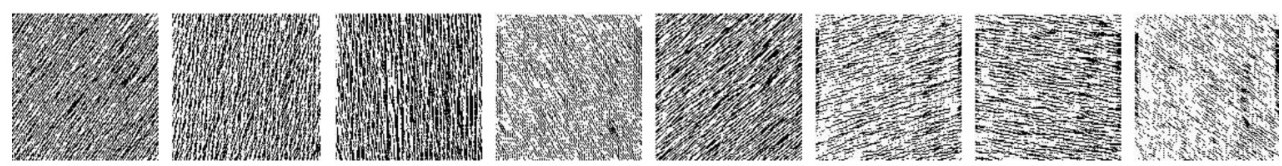

(a) The sub-bands of horizontal direction
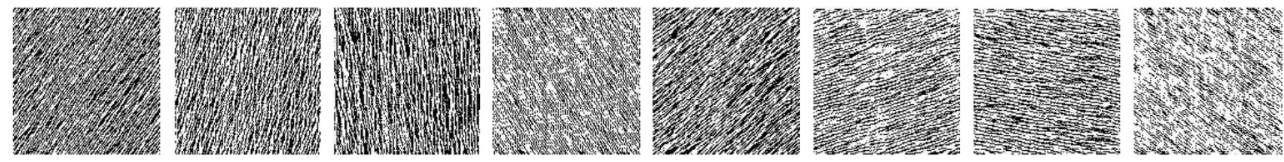

(b) The sub-bands of vertical direction
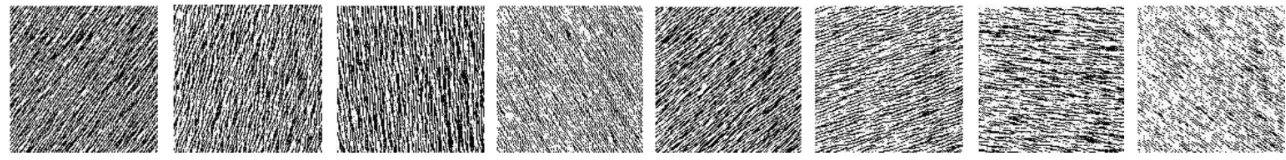

(c) The sub-bands of diagonal direction

Figure 12. 8 Directional sub-bands

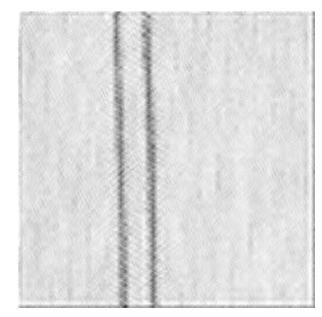

Figure 13. The reconstruction of the broken warp

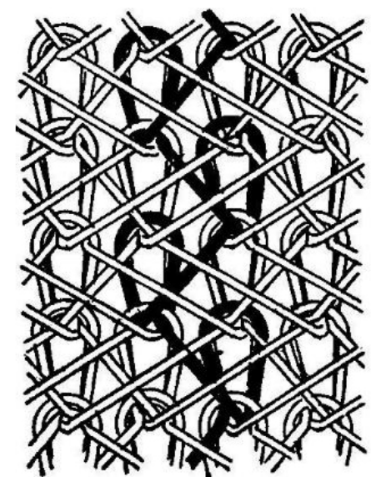

(a) the technical face

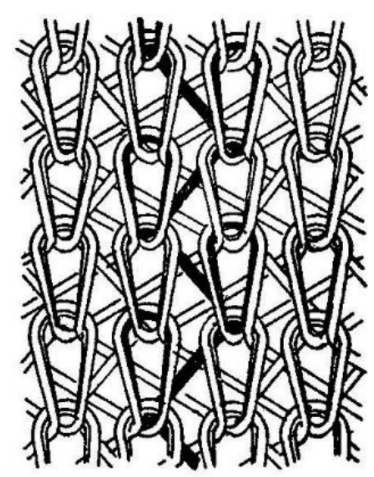

(b) the technical back

Figure 14. The loop structure of warp-knitted fabric

while smooths the large objects [30]. Accordingly, the profile of the image will not shrink through morphological opening but can be distinguished. And by experiment, the structure element in morphological opening is a disk of radius 1 . Finally the desultory points of the segmented binary image are removed. Shown in Figure 15 is the final result of the segmentation.

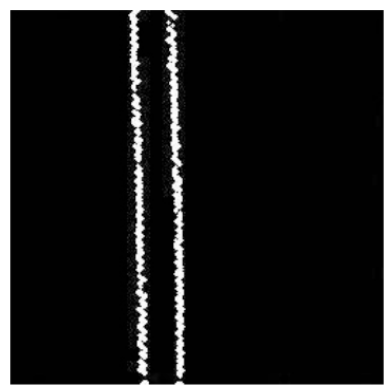

Figure 15. The final binary image

\subsection{Results and discussion}

Through the experiments, when the fundamental wave of wavelet transform and NSDFB is respectively bior2.8 and pkva6, the decomposition level is 2 and the structure element is a disk of radius 1 , NWCT can achieve the best segmentation result. It holds the most of fabric defect profile information, and the profile of the segmented defect is highly similar to the real profile. Furthermore, it can provide the reliable information for the next examination of warp-knitted fabric defect identification. Figures 16 to 21 show the results of the nondefect, broken warp 2, the width barrier 1 , the width barrier 2, the oil 1 and 2 . As for the width barrier results, these fabric defects are just like points in line while the defects are too small to distinguish from the normal hole effect. Consequently, some points of the width barrier are lost in the final results. In addition, these should be the topic of research in the further work. 


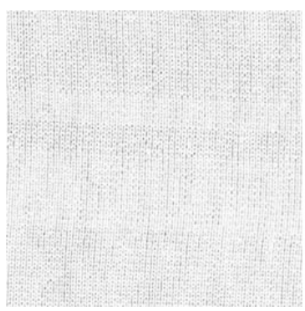

(a) Original

Figure 16. The results of the nondefect

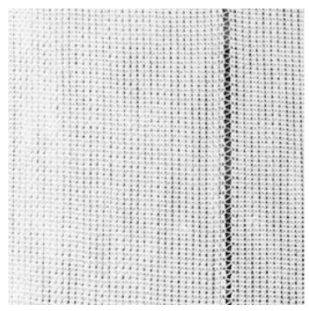

(a) Original

Figure 17. The results of the broken warp 2

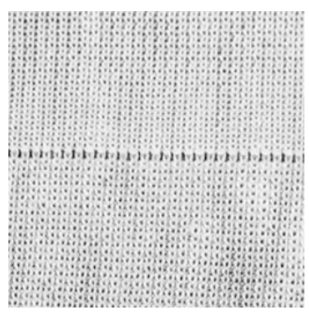

(a) Original

Figure 18. The results of the width barrier 1

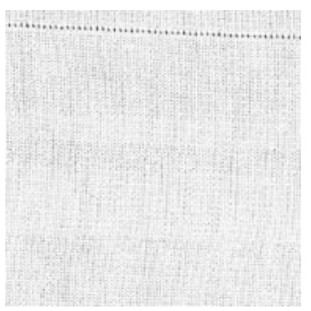

(a) Original

Figure 19. The results of the width barrier 2

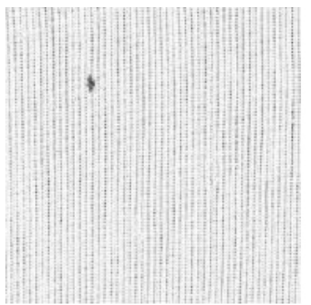

(a) Original

Figure 20. The results of the oil 1

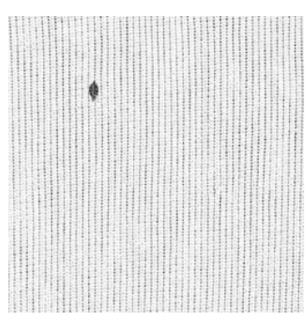

(a) Original

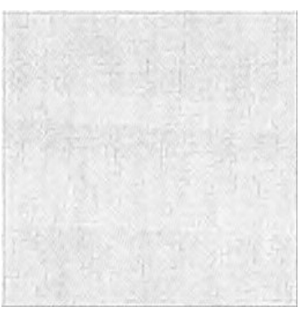

(b) reconstructed

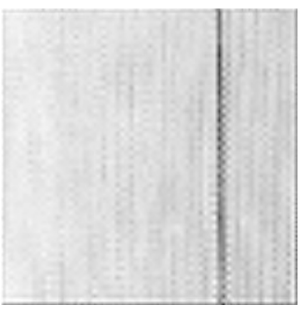

(b) reconstructed

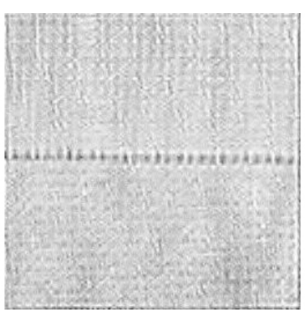

(b) reconstructed

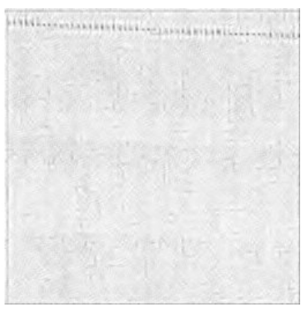

(b) reconstructed

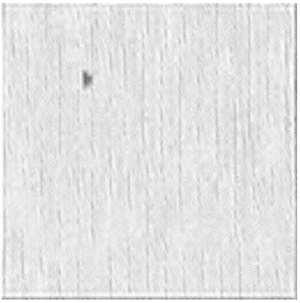

(b) reconstructed

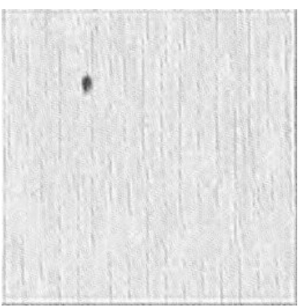

(b) reconstructed

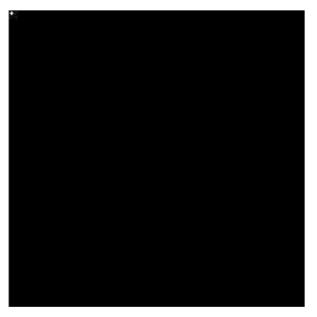

(c) result

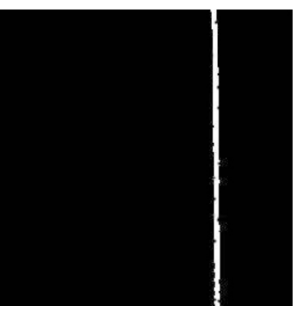

(c) result

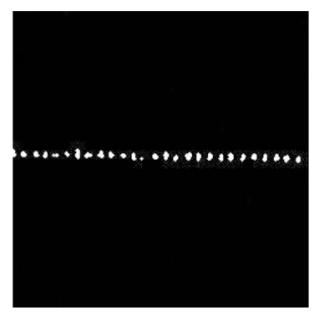

(c) result

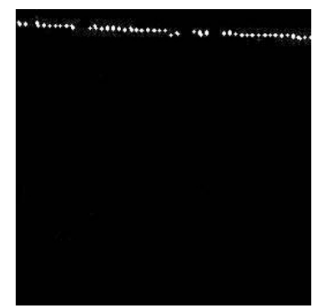

(c) result

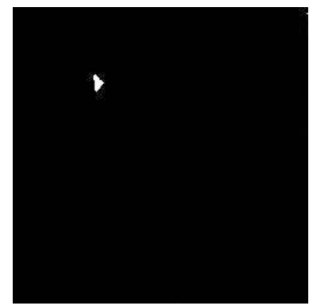

(c) result

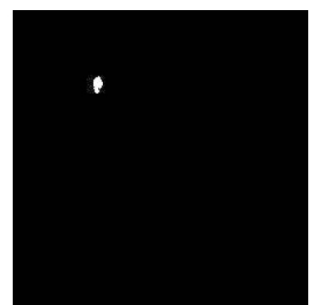

(c) result

Figure 21. The results of the oil 2 


\section{Conclusion}

This work substitutes the wavelet transform for Laplacian pyramid by the contourlet transform and constructs an improved wavelet threshold denoising method. It removes the down-sampling operation and applies the up-sampled matrix in the construction of non-subsampled directional filter bank. Consequently, the non-subsampled wavelet-based contourlet transform is formed and for the first time applied in the segmentation of warp-knitted fabric defect.

After the decomposition, the warp-knitted fabric defect image is reconstructed through selecting the promising sub-bands of the every level's high coefficients $V, H$, and $D$ based on the regional energy value. Taking the loop structure of the warp-knitted fabric into account, the reconstructed image is segmented by means of the adaptive threshold segmentation and morphological opening effectively. The profile of the fabric defect is almost intact and distinct enough so that it can be used in the next work of fabric defect identification. Further research is needed to enhance the stability of the width barrier segmentation.

\section{Acknowledgments}

The authors acknowledge the financial support from the China Postdoctoral Science Foundation (No. 2016M591767), the Fundamental Research Funds for the Central Universities (JUSRP51625B and JUSRP51404A), the Applied Foundation Research Funds of China Textile Industry Association (J201604), and the Natural Science Foundation of Jiangsu Province (No. BK20151129).

\section{References}

[1] Ngan Henry Y.T., Pang Grantham K.H., Yung Nelson H.C. (2011). Automated fabric defect detection-A review. Image and Vision Computing, 29, 442-458.

[2] Shimizu Y. (2000). Expert system to inspect fabric defects by pattern recognition. Sen-iGakkaishi, 46(1), 460-469.

[3] Tsai I.S, Lin C.H, Lin J.J. (1995). Applying an Artificial Neural Network to Pattern Recognition in Fabric Defects. Textile Research Journal, 65(3), 123-130.

[4] Haralick R.M. (1979). Statistical and structural approaches to texture. Proceedings of the IEEE, 67(5), 786-804.

[5] Ozdemir S., Ercil A. (1996). Markov random fields and Karhunen-Loeve transforms for defect inspection of textile products. Emerging Technologies and Factory Automation, EFTA '96. Proceedings. 1996 IEEE Conference on IEEE, 697-703.

[6] Cohen F.S., Fan Z., Attali S. (1991). Automatic inspection of textile fabrics using textural models. Patter Analysis and Machine Intelligence, 13(8), 803-808.

[7] Castellini C., Francini F., Longobardi G., et al. (1996). Online textile quality control using optical Fourier transforms. Optics \& Lasers in Engineering, 24(1), 19-32.

[8] Chan C-H., Pang Grantham K.H. (2000). Fabric defect detection by Fourier analysis. Industry Applications, 36(5), 1267-1276.

[9] Sansoni P., Tiribilli B. (1996). Weaving defect detection by Fourier imaging. Proceedings of SPIE - The International Society for Optical Engineering, 2786, 9-18.
[10] Escofet J., Navarro R., Millán M.S., et al. (1996). Detection of local defects in textile webs using Gabor filters. Vision Systems New Image Processing Techniques, 2785(8), 163170.

[11] Kumar A., Pang Grantham K.H. (2002). Defect detection in textured materials using Gabor filters. Industry Applications, 38(2), 425-440.

[12] Yuan S., Zheng T. (2004). Fabric defects automatic detection using Gabor filters. Intelligent Control and Automation, 2004. WCICA 2004. Fifth World Congress on. IEEE, 4, 3378-3380

[13] Tsai D.M., Bo H. (2001). Automatic surface inspection using wavelet reconstruction. Pattern Recognition, 34(6), 12851305.

[14] Tsai D.M, Chiang C.H. (2003). Automatic band selection for wavelet reconstruction in the application of defect detection. Image and Vision Computing, 21(5), 413-431.

[15] Yang X., Pang G., Yung N. (2004). Discriminative training approaches to fabric defect classification based on wavelet transform. Pattern Recognition, 37(5), 889-899.

[16] Do M.N, Vetterli M. (2003). Contourlets. Studies in Computational Mathematics, 10, 83-105.

[17] Do M.N, Vetterli M. (2003). Framing pyramids. Signal Processing, 51(9), 2329-2342.

[18] Do M.N, Vetterli M. (2005). The Contourlet transform: An efficient directional multiresolution image representation. Image Processing, 14(12), 2091-2106.

[19] Bamberger R.H, Smith M.J.T. (1992). A filter bank for the directional decomposition of images: theory and design. Signal Processing, 40(4), 882-893.

[20] Dou J., Li J. (2012). Optimal image-fusion method based on nonsubsampled contourlet transform. Optical Engineering, 51(10).

[21] Park S., Smith M.J.T, Mersereau R.M. (2004). Improved structures of maximally decimated directional filter banks for spatial image analysis. Image Processing, 13(11), 14241431.

[22] Hong P.S, Smith M.J.T. (2002). An octave-band family of nonredundant directional filter bank. Acoustics, Speech, and Signal Processing, 5, 1165-1168.

[23] Mallat S., Zhong S. (1992). Characterization of signals from multiscale edges. Pattern Analysis \& Machine Intelligence, 14(7), 710-732.

[24] Mallat S., Hwang W.L. (1992). Singularity detection and processing with wavelets. Information Theory, 38(3): 617643.

[25] Gonzalez R.C., Woods R.E. (2010). Digital Image Process, Third Edition. Pearson Education, 483-543.

[26] Eslami R., Radha H. (2005). New image transforms using hybrid wavelets and directional filter banks: analysis and design. Image Processing, 9, 733-736.

[27] Eslami R., Radha H. (2004). Wavelet-based Contourlet transform and its application to image coding. Image Processing, 5(10), 3189-3192.

[28] Cunha A.L,, Zhou J., Do M.N. (2006). The nonsubsampled contourlet transform: theory, design, and application. Image Processing, 15(10), 3089-3101.

[29] Jiang G.M. (2010). Production technology of warp-knitted fabric-warp knitting theory and typical products. First Edition. Beijing, China: Textile \& Apparel Press, 1-3.

[30] Mahajan P.M, Kolhe S.R., Patil P.M. (2009). A review of automatic fabric detection techniques. Advances in Computational Research, 1(2), 18-29. 